\title{
Adjektivphrase Im Deutschen: Eine Forschungsüberblick Über Die Struktur Der Adjektivphrase
}

\author{
Vorgelegt von M.A. Layla Esa Salim \\ Schlüsselwort: Die Adjektivphrase - Das Adjektiv-Die Phrase- Die Grammatik
}

DOI: $10.37648 /$ ijrssh.v10i04.041

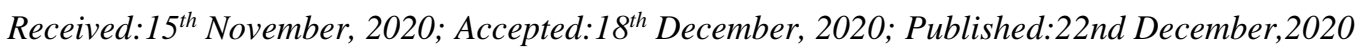

\section{DIE EINLEITUNG}

Die vorliegende Forschung lautet "Adjektivphrase im Deutschen ". Das Thema Phrase wird im vorigen Jahren von vielen Forschern und Wissenschaftlern untersucht, weil sie große Rolle in Gegenwart deutschen Grammatik und auch im Prinzip Lernen und Lehren der Deutschen Sprache spielt.

In der vorliegenden Forschung wird die Adjektive, Phrase und Hauptthema Adjektivphrase im engeren und weiten Sinne Untersucht. Das Adjektiv als eine Art von Wortarten, die flektieren kann, spielt sonderlich wichtige Rolle in der Deutschen Grammatik. Diese sonderliche und wichtige Rolle kommt von der umfassende Verwendung in der Sprache sowie die drei Arten von Flexion auch die Funktion der Adjektive in der Satzstruktur.

Das Adjektiv kommt in Verbindung mit anderen Wortarten und auch die Flexion diese Wortart beruht auf andern Wortarten. Die Phrase hat auch wichtige Stelle in der Grammatik und wie oben erwähnt im Prinzip Lernen und Lehren.

Die Phrase befindet sich auch in unterschiedlichen Arten und diese Arten beruht auf die Wortarten im Deutschen. In dieser Forschung wird die Adjektivphrase ausgewählt, da diese Art wichtig Rolle im Deutschen hat.

Die Forschung besteht aus zwei Abschnitten. Der erste Abschnitt schließt die Definition der Adjektive, Merkmale der Adjektive, Formen der Adjektive, Zum Gebrauch des Adjektivs im Satz, Die Aufgaben des Adjektivs, Die semantischen Aspekt des Adjektivs, Die grammatischen Eigenschaften des Adjektivs, Klassifizierung des Adjektivs nach der Bedeutung, Deklinationsformen des Adjektivs ein. Der zweite Abschnitt enthält die Phrase, die Phrase im Deutschen, Arten der Phrase, Kern der Phrase, Adjektivphrase sowie Schlussfolgerung Am Ende findet die Literaturangaben.

\section{DIE DEFINITION DES ADJECTIVES}

Die Adjektive definiert als “ ....... Eigenschaftswörter, Wiewörter genannt“. (George 1995:92). Eine andere Definition von Wahrig wird wie im Folgenden:

Adjektiv: "Ad.jek.tiv, Ad.jek.ti.vum ein Wort, das ein Substantiv oder Verb nähe bestimmt u. dekliniert werden kann; Beiwort, Eigenschaftswort und kommt aus dem Latein Adiectio „Zusatz oder zu etwas fügen" (Wahrig 2005:40)

Angesichts der erwähnten Definitionen, wird es versteht, dass das Adjektiv ein beschreibendes Wort, Eigenschaftswort, Bezugsname oder ein Verb näher festlegt wird und es flektieren werden kann nach den Substantiven. Die Deklination der Adjektive kann stark oder schwach oder gemischt sein. 
Davon gehen wir aus, dass das Adjektiv eine Wortart und deklinierbar ist. Adjektiv beschreibt die Nomen. Es gibt auch den Fall bei der, dass ein Substantiv oder Verb zu einem Adjektiv verändert wird, z.B.: Ernst- ernst [ $\rightarrow$ Adjektiv] dieses Prozess heißt adjektivieren (Wahrig 2005:64).

Andererseits nach der Definition von Duden werden die Adjektive nach einigen detaillierten definiert. Es wird die Eigenschaften veranschaulicht:

"Adjektive unterscheiden sich von den anderen Wortarten nicht nur in der Flexion, sondern Syntaktisch. So können nur Adjektive zwischen definitem Artikel und Substantiv stehen. Diese Beobachtung lässt sich für eine Probe ausnutzen: Wörter, die zwischen definitem Artikel und Substantiv stehen können, sind Adjektive" (Duden 2005:457)

Nun ist es zu zeigen, welche Feststellungen von dieser Definition verstehen können,: In Bezug auf Flexion und Syntax unterscheiden sich Adjektive von anderen Wortarten. Für die Adjektive erscheint die Möglichkeit, dass sie zwischen definitem Artikel und Substantiv stellen. Es wird diese Erklärung mit folgenden Beispielen unterstützt:

(a) Arica kehrt sicher wieder.

$\rightarrow$ die sichere Wiederkehr $\rightarrow$ können also Adjektiv sein

(b) Arica kehrt vielleicht wieder

$\rightarrow$ die* vielleichte Rückwieder $\rightarrow$ hier nicht als Adjektiv, aber andere Wortart (Adverb)

\subsection{Merkmale der Adjektive}

Adjektive bestimmen sich durch folgende Merkmale(George 1995:92):

- Sie treten vor Substativen auf und werden dann von diesen regiert. Sie können als Bauteile von Wortgruppen in einem Satz mit einem Nomen:

ein wunderbares Geschenk

- Sie sind flektierbar:

ein kleiner Fleck, eines kleinen Fleckes, ein kleines Mädchen

- sie können kompariert werden. (Steigerung):

schwach, schwacher, am schwächsten

- sie haben gelegentlich ein direktes Gegenteil: schön - hässlich, groß - klein, jung- alt

- Sie können in Stufen abteilen bzw. graduiert werden : wirklich gut, recht gut

\subsection{Die Formen der Adjektive}

Die Adjektive nach George (George 1995:92) werden in drei Formen unterscheidet

- Adjektive, die geborene heißt:

gut, alt, groß, schön, lang, ganz, klein, fertig, jung, recht, weit, schnell

- Adjektive, die als Partizipien sind:

wütend, brüllend, entscheidend; entschieden, verwirrt, verrückt; auch z.B. geteiltes Land, versalzenes Essen, entschwundene Hoffnung.

- Adjektive, die abgeleitet werden, und heißt abgeleitete Adjektive:

Die kommt aus Adjektiven (rötlich, reichlich, fröhlich, unklug, dreifach); die kommt aus Verben (heizbar, erklärlich, spendabel, unaufhörlich, durchlässig, schmeichelhaft); die kommt aus Substantiven (polizeilich, römisch, sandig, eisern, fleischlos); die kommt aus Adverbien (morgig, ehemalig, sonstig) 


\subsection{Zum Gebrauch des Adjektivs im Satz}

Die Adjektive allerdings spielt eine große Rolle im deutschen Satz. Die gespielte Rolle des Adjektivs lautet folgendermaßen (George 1995:93):

(a) Als Attributiv:

ein dünner Hahn, von rötlicher Gattung, ein maßgebendes Bescheid

Sie bilden mit Substantiven eine Einheit und stehen vor ihnen: sie tragen die gleichen Kasus wie das Substativ und wird flektierbar. Es wird erwähnt, dass das attributive Adjektiv vor einem substantivischen Kompositum steht, bezieht es sich dann auf Inhalt der ganzen Verbindung. Deshalb sollte man kein attribuierte Kompositum steht, dass das Adjektiv lediglich auf das bestimmte Grundwort hindeutet, wie folgt (Duden 2005:347):

*tiefgekühlte Dosenfutter (stattdessen: das Futter mit tiefgekühlten Dosen)

(b) Als Prädikativ:

Das Adjektiv ist in diesem Fall zum deklinierten Verb angehörig, es unterlegt nicht den Regeln der Deklination und erklärt etwas über das Subjekt. z.B.:

Paul ist dünn, Layla liegt schläfrig im Sofa

(c) Als Adverbal ( das Verbrelevant):

Mihaela rennt schnell, der Ansager spricht laut

Das Adjektiv ist in diesem Kasus zum flektierten Verb angehörig, es wirst nicht flektiert und erklärt etwas über die verbalen Geschehen. Man kann hier beobachten, dass das Adjektiv im adverbalen Gebrauch auf das Verb bezieht, aber im prädikativen Gebrauch wird eine Eigenschaft zur Person oder Gegenstand zugeschrieben. Diese beiden Gebräuche werden deutlich durch folgende Beispiele:

- prädikativ: Paul ist dünn - der dünne Paul

- adverbal: Mihaela rennt schnell - ihr schneller Rennen

- adverbial (verwendet wie ein Adverb):

weit vorwärts, unbewusst bescheidend, kurz nach sieben

In diesem Fall Wörter anderer Wortart werden durch Adjektive modifiziert: Adverbien, Adjektive, Präpositionen. Gleiche Gliederung zur Leistung des Adjektivs wird (Duden 2005:347) wie folgt gestellt:

- attributiver Gebrauch

- nominalisierter Gebrauch

- Prädikativer Gebrauch

- adverbialer Gebrauch

Im Duden Band 4 die Adjektive, die keine Endung haben, wird in der Regel nicht flektiert; "der Grundsatz dafür legt" Ihr Stamm geht dann überwiegend (a) auf einen Konsonanten oder (b) auf einen Vollvokal aus" (Duden 2005:362) wie folgt:

(a) Groß, rot, seltsam, hart, übel

(b) Prima, treu, tabu

Nach Duden Band 4; Adjektive, die als attributiv und prädikativ gebraucht, lassen sich überlagern. Der Grund dafür ist: “ wenn ein Adjektiv Bestandteil einer prädikativen Nominalphrase ist, deren Substantiv eingespart worden ist“" (Duden 2005:358), man kann das tatsächlich wie folgt vorliegen:

Dieses Buch ist ein nützliches (= ein nützlich Buch). 


\subsection{Die Aufgaben des Adjektivs}

Das Adjektiv wie oben erwähnt wird, fungiert stilistisch eine von den wichtigsten Elementen im Deutschen, kürzlich bietet diese Aufgabe mit einem Beispiel ${ }^{1}$ :

1. Klassifizieren:

Es gibt hier hübsche, elegante, liebvolle und klügste Frauen.

2. Unterscheiden:

Die junge Frau mit den weiten, ohne faltigen Augen; die alte Frau mit den kleinen, faltigen Augen

3. Beschreiben:

Wir laufen ziemlich fleißig; braune, grobe Steine.

4. Charakterisieren:

Ihr ironisches Kritik

5. Schmücken:

aus weichender, seid durchwirkter Stoff; silbergrau strahlende Papiere

6. Werten:

ein nettes, liebenswürdiges Mädchen

sie findet ihnen leise

Gemäß was oben erwähnt würde, schließt man auf den ersten Blick, dass Adjektive unwichtig lauten können. Sie sind hier abhängig lediglich auf begleitende Worte von Verben und Substantive, dem es untergeordnet ist; Es hängt auf was der Schreiber will, zwingt ihn das gerade zur Sorgsamkeit auf. Vor allem der Fall bei allgemeinen Substativen:

persönliche Arbeit, der sportwissenschaftliche Bereich, schlechte Worte, ihre geschäftliche Bedürfnisse

In diesem Zusammenhang erschließen können, das Adjektiv zu Art und Weise des Textes passen sollte und es stilistisch und inhaltlich im Gegensatz zu seinem Referenzwort sein sollte.

\subsection{Die semantischen Aspekt des Adjektivs}

\section{Die semantische Adjektivabfolge}

Von Anfang und damit diese Abfolge des Adjektivs semantisch besser zu verstehen, was im Duden Erklärung (Duden 2006:346) die beste demnach wäre.

"Adjektive leisten semantisch Unterschiedliches. Viele ordnen einer Person oder Sache eine Eigenschaft zu, man spricht dann von qualifizierenden Adjektiven. Andere drücken eine Beziehung oder Zugehörigkeit aus; man spricht dann von relationalen Adjektiven"

Eisenberg und andere Autoren schlagen irgendwie ähnliche Adjektivabfolgen vor: a. quantifizierende Adjektive, relative pronominale Adjektive des Ortes und der Zeit, Wertungswörter im weiteren Sinn, Bezeichnung verschiedener Relationen, phraseologisch gebundene Adjektive; b. quantifizierende, referentielle, qualifizierende, einordnende Adjektive oder c. quantitative, referentielle, qualitative, Herkunftsadjektive und klassifikatorische Adjektive (Eisenberg 1999: 405).

${ }^{1} \mathrm{Vgl}$. nach George 1995:105 
All diese Klassifikationen ihren Anfang haben im ersten Behaghelschen Gesetz, hiermit „,das geistig eng Zusammengehörige auch eng zusammengestellt wird (Behaghel 1932: 4, Eisenberg 1999: 405).

Zusammenfassend versteht man daraus, dass Adjektivleistung semantisch ist unterschiedlich erweisend. Manche von ihnen reihen eine Eigenschaft einer Person oder Sache ein, hier spricht man von Adjektiven, die qualifizierende Eigenschaften haben.

Andere Teile die sind relationalen Adjektiven, sie drücken eine Zugehörigkeit oder Beziehung aus. Dazu gibt es Zahladjektive, die als Teil von quantifizierenden Adjektiven fungieren (Duden 2005:346-456).

Schließlich wie wir bereits erwähnt die als besondere Fälle, die adjektivisch gebrauchten Partizipien. Während die adverbial gebrauchten Adjektive als eigenständige Satzglieder fungieren, wie Duden aufführen.

Im folgenden Satz sollte man formal zu unterscheiden, welche sichtbare Ableitungen sind; der Grund dafür ist:“ Zumindest formal gibt es keinen Grund, anzunehmen, dass auch im Deutschen ein (unsichtbarer Wortartwechsel vorliegt" (Duden 2005:360) bzw. zum Wortart Adverb werden adverbial gebrauchte Adjektiv grammatisch nicht zugeordnet:

Glücklicherweise ist sie gekommen:

In der folgendem Satz verhält sich Deutsch wie Englisch:

Fortunately, she came.

\subsection{Die grammatischen Eigenschaften des Adjektivs}

Nach Duden die grammatischen Eigenschaften des Adjektivs können mit drei folgende Eigenschaften zusammenfassen (Duden 2005:345-456):

- Sie können verändert werden, also das hängt von drei Fällen, die sind, nach dem Fall der Kasus, nach dem grammatischen Zahl, und nach dem grammatischen Geschlecht. z.B. die Substantive und Pronomen und anders hängt nicht auf festes Genus; im Gegenteil sie treten in allen drei Gattungen auf:

Maskulinum: ein satirischer Ratschlag

Femininum: eine satirische Empfehlung

Neutrum: ein satirisches Empfinden

Sie können aber ohne deklinierte Form zu kommen:

Dein Vorschlag war satirisch

- Bezüglich das Flektieren des Adjektivs gibt es zwei Typen der Endungen, die sind, starke und schwache Flexion:

Stark: $\quad$ ein warmer Tee

Schwach: der warme Tee

- Adjektivbildung können meistens als die Komparativformen werden:

Positiv: warm das moderne System

Komparativ: warmer das modernere System

Superlativ: am wärmsten das moderneste System

Nach H. Leiß kann man die Folgenden erschließen: „"Die Komparation ist sowohl bei undeklinierten wie bei deklinierten Formen des Adjektivs möglich,, (Leiß 1998:26):

Das ist klug - kluger - am klügsten “ bzw. die kluge - klügere - klügste Bemerkung 


\subsubsection{Partizipien als Adjektive}

In diesem Kontext können wir auf Leiß Erläuterung angewiesen sein (Leiß 1998:20):

"Darunter fallen die nominalisierten Adjektive wie der/die/das Schöne und entsprechend die nominalisierten Partizipien wie der/die Betrogene, sowie einige ähnlich gebildete Nomina wie der Beamte (statt der Beamtete). Sie können nach den verschiedenen Deklinationstypen des Adjektivs dekliniert werden".

Nach dieser Zitat versteht man darunter, dass diese Art von Partizipien wie Adjektive verhält werden. Wir meinen damit die Verwendung des mehrteiligen Begriffs ,adjektivische gebrauchten Partizipien“:

das umbaute Krankenhaus, ein erzitternder Blatt, die zu befindenden Personen

Einen Grundsatz betreffend gilt die folgende Darstellung auch als Adjektiv gebrauchtbaren Partizipien. Sie liegt unter besonderen Beschränkungen, wird das für jeden Fall später angegeben. Also als Adjektiv gebrauchtbaren Partizipien lassen sich nicht kompariert werden.

der brüllende Hahn $\rightarrow$ der* brüllendere Hahn (Bestenfalls anstelle: der lauter/ heftiger brüllende Hahn)

Dazu hinzufügen auch, dass Partizipien als unabhängige Adjektive wirken können, dann die Beschränkungen für Partizipien nicht mehr gültig sein:

das anziehende Mädchen $\rightarrow$ das anziehendere Mädchen, das anziehendste Mädchen

Aber es befindet sich auch Ausnahmefälle:

„“Grundsätzlich können alle Adjektive gesteigert werden, die attributiv verwendbar sind. Partizipien werden nur gesteigert" (George 1995:96). Im Prinzip können alle Adjektive kompariert werden, die als Attributiv geeignet zu verwenden. Partizipien, die schon als Adjektiv aufnehmen sind, werden ausschließlich gesteigert (Vgl. George 1995:96)

Das beweinteste Mädchen, die zwängte Leitung

Aber kaum sein:

die lachelndere Frau, der eignenere Anzug

Die zweiten Teile der zusammengesetzten Adjektive werden auch wie normale Adjektive gesteigert:

altägyptisch - altägyptischer - altägyptischst

übelstand - übelstander - übelstandst

vielbeschäftigt - vielbeschäftigter - vielbeschäftigtest

Gibt es anderer Möglichkeit bei zusammengesetzten Prinzipien, dass der erste oder der zweite Teil von ihnen gesteigert werden kann:

umfassendere/umfassende Verwaltungsakt

\subsection{Klassifizierung des Adjektivs nach der Bedeutung}

Des Weiteren werden die folgenden Arten von Adjektiven nach ihrer Bedeutung katalogisieren (Vgl. George 1995:94):

- Beiwörter oder Eigenschaftswörter, die auf Merkmale und Eigenarten von Gegenständen weisen: z.B. schwarz, heiß, grob.

- Orientierungswörter, die man räumlich oder zeitlich einordnen können z.B. gegenwärtig, derzeitig, lokal örtlich

- Bewertungswörter, die nach denen wir Gegenstände werten z.B. schlecht, hässlich, nutzbar, kindlich.

- Anzahlwörter, die auch Kardinalzahlen bezeichnen, beziehen sich auf Anzahle z.B. drei, fünf, sieben, tausend. In diesem Zusammenhang erklären wir, dass diese Numeralia außer ein, zwei, drei- nicht flektiert werden, während die Ordinalzahlwörter, die auf Ordnung hinweisen, werden wie andere Adjektive flektiert wie dritte, vierte, hunderte, zwanzigste.

Diese Art von Wörtern können auch in Substantiven umgewandelt werden:

Er ist jetzt Ende Dreißig. 
Wer heiratet seine Geliebte am Ersten? das zweite Hundert.

Verweiswörter wie letztere, sonstig, o.g., nachfolgend, nächste, andere, derartig, sie stellen genannte Informationen im Textteile her, o. Ä. die im vorliegenden Kontext nachzulesen wird.

Diesbezüglich machen wir deutlich, dass die Wörter ersterer und letzterer nur verwendet sollen, wenn wir den Verweis auf grammatisch gleiche Personen oder Dinge klarstellen möchten:

Diana hat ein Auto und Fahrrad. Letzteres benutzte sie fast täglich.

Ersteres war neu.

Aber können nicht sagen:

Das Skateboard ist im Kellerraum. Letzterer war lange nicht geputzt.

\subsection{Deklinationsformen des Adjektivs}

Es gibt für jede attributive Adjektive zwei unterschiedliche Formen von Deklination, die sind schwachem und starkem Form. Die schwächste nimmt fast den gleichen Endungen (n-) des Substantivs. Alle Formen haben die gleiche Beugung und sind kaum unterscheidet. Siehe die nächste Tabelle (George 1995:100):

\section{Form die schwache Deklination}

\begin{tabular}{|c|c|}
\hline Singular & Plural \\
\hline $\begin{array}{l}\text { der } \rightarrow \text { wunderbare } \rightarrow \text { Student } \\
\text { die } \rightarrow \text { wunderbare } \rightarrow \text { Frau } \\
\text { das } \rightarrow \text { wunderbare } \rightarrow \text { Mädchen }\end{array}$ & $\begin{array}{c}\text { die } \rightarrow \text { wunderbaren } \rightarrow \text { Studenten } \\
\text { die } \rightarrow \text { wunderbaren } \rightarrow \text { Frauen } \\
\text { die } \rightarrow \text { wunderbaren } \rightarrow \text { Mädchens }\end{array}$ \\
\hline $\begin{array}{l}\text { des } \rightarrow \text { wunderbaren } \rightarrow \text { Studenten } \\
\text { der } \rightarrow \text { wunderbaren } \rightarrow \text { Frau } \\
\text { des } \rightarrow \text { wunderbaren } \rightarrow \text { Mädchens }\end{array}$ & $\begin{array}{l}\text { der } \rightarrow \text { wunderbaren } \rightarrow \text { Studenten } \\
\text { der } \rightarrow \text { wunderbaren } \rightarrow \text { Frauen } \\
\text { der } \rightarrow \text { wunderbaren } \rightarrow \text { Mädchens }\end{array}$ \\
\hline $\begin{array}{l}\text { dem } \rightarrow \text { wunderbaren } \rightarrow \text { Student } \\
\text { der } \rightarrow \text { wunderbaren } \rightarrow \text { Frau } \\
\text { dem } \rightarrow \text { wunderbaren } \rightarrow \text { Mädchen }\end{array}$ & $\begin{array}{l}\text { den } \rightarrow \text { wunderbaren } \rightarrow \text { Studenten } \\
\text { den } \rightarrow \text { wunderbaren } \rightarrow \text { Frauen } \\
\text { den } \rightarrow \text { wunderbaren } \rightarrow \text { Mädchens }\end{array}$ \\
\hline $\begin{array}{l}\text { den } \rightarrow \text { wunderbaren }- \text { Student } \\
\text { die } \rightarrow \text { wunderbare } \rightarrow \text { Frau } \\
\text { das } \rightarrow \text { wunderbare } \rightarrow \text { Mädchen }\end{array}$ & $\begin{array}{c}\text { die } \rightarrow \text { wunderbaren } \rightarrow \text { Studenten } \\
\text { die } \rightarrow \text { wunderbaren } \rightarrow \text { Frauen } \\
\text { die } \rightarrow \text { wunderbaren } \rightarrow \text { Mädchens }\end{array}$ \\
\hline
\end{tabular}

- unflektierte Adjektive: berufen sich die unflektierte, anders modifizierte Adjektiv auf Substantiv, sie werden durch Komma getrennt und parallel flektiert (George 1995:102):

modifizierende Form

ein scheußlich kalter Wind

brennend heißer Wüstensand

ein gähnend langweiliger Mensch parallele Form

ein scheußlicher, kalter Wind

brennender, heißer Wüstensand

ein gähnender, langweiliger Mensch

Die ähnliche Meinung hat (Duden 2005:351-468) im Bereich unflektierte Adjektive in lockeren Nachträgen:

Allgemein üblich sind unflektierte Adjektiv, die dem Substantiv als lockerer Nachtrag folgen. In geschriebener Sprache werden sie dann in Kommas eingeschlossen. 
Nach dieser Regel haben beide Adjektive sowohl modifizierend als auch parallel andersartige Bedeutung. Hier die Modifizierende Form des Adjektiv kommt als adverbial (wird kalt durch das Gebrauch adverbial scheußlich: in scheußlich kalt näher festlegen) auf der anderen Seite in scheußlicher, kalter Wind: hier Wind zeigt an, dass er scheußlich lautet.

\subsection{Gesteigerte Formen des Adjektivs}

Nach George: „Adjektive haben neben der Grundform gesteigerte Formen oder Vergleichsformen“ (George 1995:95) Das heißt neben der Hauptform des Adjektivs gibt es auch gesteigert Adjektive oder was genannten Vergleichsformen. Diese Formen kann man verwendet, wenn man Eigenschaften der Gegenstände oder Personen vergleicht:

- Positivstufe (-Grund): langsam, die langsame Schildkröte

- Komparativstufe (-Höher): langsamer, die langsamere Schildkröte

- Superlativstufe (-Höchste): am langsamsten, die langsamste Schildkröte

Notiz: Die Form (am +-sten) wird als nicht Attributiv gebildet.

Neben dem regelmäßig Anhängen des Komparativs mit -er und Superlativs mit -(e)st, kommt hinzu der Stammvokal der umgelautete einsilbige Adjektive, wie folgt im Tabelle der Komparation (George 1995:95):

\begin{tabular}{|l|l|l|}
\hline Positiv & Komparativ & Superlativ \\
\hline $\begin{array}{l}\text { groß } \\
\text { hart }\end{array}$ & größer & $\begin{array}{l}\text { am größten } \\
\text { am härtesten }\end{array}$ \\
& härter & am klügsten \\
& klüger & \\
\hline
\end{tabular}

Nach Duden Band 4 erwähnt wird, dass Adjektive, die auf Betonung der Attributive Gebrauch bedient, erzielt die gleichen Zweck:" Die Betonung der attributiv stehenden erfüllt oft den gleichen Zweck“(Duden 2005:359), Anschaulicht wird wie folgt:

das ist ein öffentliches (kein individuell) Leid.

\section{Ersatzformen:}

Es gibt auch einige Adjektive, die unregelmäßige gesteigerte Formen haben, wie folgt in der Tabelle auf unregelmäßige Komparation (George 1995:96):

\begin{tabular}{|l|l|l|}
\hline Positiv & Komparativ & Superlativ \\
\hline $\begin{array}{l}\text { arg } \\
\text { dumm } \\
\text { nass } \\
\text { wild }\end{array}$ & ärger & $\begin{array}{l}\text { am ärgsten } \\
\text { am dümmsten } \\
\text { am nassesten } \\
\text { am wildesten }\end{array}$ \\
& nasser & \\
\hline
\end{tabular}

Auch bei unregelmäßige Adjektive bei der auf -el endet, fällt das -e im Komparativ, aber im Superlative wird regelmäßig: eitel - eitler - am eitelsten

heikel - heikler - am heikelsten dunkel - dunkler - am dunkelstem 
Bei Adjektive mit -er endet, wird das -e auch weggelassen, wenn bevor -er ein Diphthong kommt:

sauer - saurer - sauerste

teuer - teurer - teuerste

Was übrig bleiben von Adjektiven, die mit Suffix - er und -en endet, haben Doppelformen:

basalten - basaltener/basaltner - basaltenste

birken - birkener/birkner - birkenste

Man findet auch Ausnahmefälle:

Im Buch „, Grammatik und Stil für George,, wird Sonderfalle in diesem Kontext gefunden und erklärt als „" Sind die beiden Teile noch nicht so eng zusammengewachsen, kann nur erste Teil gesteigert werden“ (George 1995:96). Das heißt; wenn die beiden Teile kein enge Zusammenwachsen haben, kann nur in diesem Fall die erste Teil gesteigert: die besserbezahlte Bank.

Außerdem gibt es geradezu bestimmte zusammengesetzte Adjektive, die einen gesteigerten Teil enthalten: weitestmöglich, hochkarätig, höchstmöglich, meistbenutzend

Es ist nicht notwendig die folgenden Adjektive noch einmal zu steigern. Also nicht:

bestbehütest, weitestmöglichst, höchstmöglichst, hochkarätigst usw.

(aber geläufig ist: die erstbeste)

Auch: bei Zusammensetzung ist nur einen Teil steigbar!

Nur bei der Substantivierung des Adjektivs sollte man auch eine Steigerung wählen.

Also die Steigerung ist nicht gedoppelt:

Das am meisten Gelegene (nicht: Gelegenste)

\section{DIE PHRASEN IM DEUTSCHEN}

Die Herkunft des Begriffs Phrase ist Griechisch. Es meinte als leere Redensart, Floskel, die nicht besagt, Beispiel (dwds.de.wb/phrase):

Eine abgeschmackte, hohle, nichtssagende, verlogene Phrase

Die Phrasen:"( auch Wortgruppen genannt) sind Gruppen von Wörtern, die nach grammatischen Regeln verbunden sind, so dass sie einen Sinneinheit bilden" (Heringer 1989:198).

Im „Deutsches Wörterbuch,, von Wahrig befindet sich nicht das Wort Phrase, sondern das Idiom und definiert als "Wort oder feste Wortgruppe, wobei die Bedeutung auf einem Bild oder einer anderen nicht wörtlich Interpretation beruht" (Wahrig 2005:64). Davon gehen wir aus, dass die Phrasen als Zwischenglieder zwischen Wort und Satz erscheint. Sie wird als quasi Fertigbauteile gegolten.

\subsection{Die Definition der Phrase}

Ein Wort oder Wortgruppe muss festgelegte Merkmale haben, damit eine Phrase zu nennen. Die Phrasen sind durch folgende Merkmale gekennzeichnet: (Herniger 1989:198)

- Phrasen bestehen (meistens) aus mehreren Wörtern. 
- Phrasen sind Bedeutungseinheiten. Beim Sprechen kann man zwischen den Phrasen Luft holen, beim Lesen kann man im Geiste verharren.

- Phrasen lagern sich um einen Kern, dem die übrigen Wörter der Phrase untergeordnet sind.

- Phrasen sind gewöhnlich zusammenhängende Ausschnitte aus Sätzen, d.h., die Wörter einer Phrase stehen eng beieinander.

- Phrasen haben feste Baupläne; die Grammatik gibt Regeln dafür, wie Phrasen aufgebaut sein können.

Angesichts dieser Darstellung konnte resultiert werden, dass die Phrase aus einem Wort oder Wortgruppe bestehen kann, hat eine Bedeutung und Kern. Der Kern der Phrase wird die Art der Phrase bestimmt.

Im Deutschen wird Arten von Phrasen gesehen. Diese werden sich durch den Innenbau der Phrase variieren. Die Arten der Phrasen unterscheiden sich von einem Sprachwissenschaftler zu anderem.

Die Arten sind im Folgenden zusammengestellt:

Nach Heriger wird drei Arten gefunden ('Heringer 1989:198):

(Verbalphrasen, Nominalphrasen und Adjektivphrasen)

Nach Kürschner gibt es folgende Phrasentypen (Kürschner 2003:160):

(Verbphrase, Substantivphrase, Pronominalphrase (Beide Nominalphrasn), Artikelphrasen, Adjektivphrase, Partikelphrase, Präpositionalphrase, Konjunktionalphrase, Interjektionalphrasen, Nebensatz, Infinitiv, Teilsatz, Partizip, Teilsatz Phrasen)

Nach Duden werden Phrasenarten in sieben Gruppen eingeteilt (Duden 2006:806): Es sind:

(Nominalphrasen, Artikelphrasen, Adjektivphrasen, Adverbphrasen, Präpositionalphrasen, Konjunktionalphrase und Verbalphrase).

\subsection{Der Kern der Phrase}

Wie oben erwähnt wird, dass jede Phrase ein Kern hat und dieser Kern gehört eine Wortart. Der Grund dafür ist: " Jede Phrase hat einen Kern. Der Kern gehört einer der Hauptwortarten an"(Heringer 1989:198).

Bestätigte das irgendwie vielmehr ähnlich Leiß wie folgt " Damit sind Adjektivphrasen gemeint, die ein undekliniertes Adjektiv oder Partizip als Kern enthalten und sich auf die Subjekt- oder Objektnominalphrasen oder das Verb beziehen kann`(Leiß: Syntax 1. 1998:41)

\begin{tabular}{|l|l|l|}
\hline Phrase & Kern & Beispiel \\
\hline Nominalphrase (NP) & Substantiv (Nomen) & Otto liebt süße Schleckereien \\
& Substantivierung \\
& Pronomen & $\begin{array}{l}\text { Otto liebt Süßes } \\
\text { Otto liebt das }\end{array}$ \\
\hline Artikelphrase (Artp) & Artikelwort & Ein Haus irgend so ein Haus \\
& & Das Haus, mein Haus, welches Haus, jedes Haus \\
\hline Adjektiphrase (Ap) & Adjektiv & Der Kellner war aufmerksam \\
& & Das ist ein ganz besonderes aufmerksamer Kellner \\
\hline Adverbphrase (AdvP) & Adverb & Anna stand vorn. Die junge Frau ganz vorn ist Anna \\
\hline
\end{tabular}




\begin{tabular}{|l|l|l|}
\hline Präpostionalphrase (PP) & Präposition & $\begin{array}{l}\text { Die Taube flog auf das Dach ich kümere mich um die } \\
\text { Sache. Direkt über uns war eine schwarze Wolke }\end{array}$ \\
\hline $\begin{array}{l}\text { Kunjunktionalphrase } \\
\text { (KonP) }\end{array}$ & (beiordende) Konjunktion & $\begin{array}{l}\text { Er verhielt sich wie ein Gentleman. Als gute Rechnerin } \\
\text { bemerkte sie den Fehler sofort }\end{array}$ \\
\hline
\end{tabular}

Eine Tabelle wird ebenso das Ergebnis aufgeführt:

Tabelle (1) (Duden 2006:806)

Die Tabelle wurde die Phrasearten durch den Kern bestimmt sowie wie jede Art auf die Wortarten beruht wird.

\subsection{Adjektivphrase}

Der Kopf oder Kern einer Adjektivphrase ist ein attributiv, adverbial oder Prädikativ verwendetes Adjektiv. Die Erweiterung des Kopfs kann die Adjektivphrase auf unterschiedliche Art und Weise aufgebaut werden. Nach (grammis.ids-mannheim), es gibt bei Adjektivphrase ein Sonderfall, es ist die Partizipialphrase bzw. diese Art von Phrasen haben ein Kopf, der entweder ein Partizip I oder ein Partizip II. George definiert Adjektivphrase wie im Folgenden (George 1995:244 - 298):

"Adjektivphrase haben ein Adjektiv als Kern, der hier hervorgehoben ist. Oft bestehen sie nur aus diesem Kern, aber grundsätzlich sind unterschiedliche Erweiterungen möglich"

Eine andere Erklärung von Leiß (Leiß 1998 :116):

Bei zusammengesetzten Adjektivphrasen werden die Bestandteile (eines Vergleichs oder Gegensatzes) getrennt:

Gleich lindert sich deine Verzweiflung, dass du keine Ferien mehr findest, die himmlisch sind statt touristisch.

Das meint, bei Adjektivphrasen, die zusammengesetzt werden, werden die Bauteile des Satzes im Fall des Vergleichs bzw. Kontrasts abgetrennt, wie folgt:

Gleich abbaut sich deine Stress, dass du keinen Erlaub mehr findest, die vergnügt sind statt unangenehm.

\subsubsection{Adjektiv als Wortarten}

Darüber hinaus für mehr Erleichterung bezüglich Wortart des Adjektiv in Hand stehen, formulierte ich die folgende Tabelle von Adjektivwortarten Fields nach "H. Leiß Tabelle 1.3: Adjektiv als Wortarten des Deutschen, S:19“.

\begin{tabular}{|l|l|l|l|}
\hline Flexionsarten & Hauptformen & Dimensionen der Form \\
\hline Deklination & & Genus, Numerus, Kasus \\
\hline Komparation & positiv, & \\
\hline & komparativ, & \\
\hline Deklinationstyp & superlativ & \\
\hline
\end{tabular}




\subsection{Die Funktionen und Arten der Adjektivphrasen}

Adjektivphrasen wird hier erklärt, die Adjektive als Wesen haben, wird dann ein hervorgehobenes Wesen. Öfter sie nur von diesem Wesen entstehen, aber prinzipiell können verschiedene Erweiterungen möglich sein. Dazu nach " Adjektiverweiterungen enthalten ganze Phrasen vor dem Adjektiv" (Grammatik und Stil 2005:259-303):

ein [äußerst netter und aktiver] Lehrer

Es war doch [sichtbar kalt].

Wer wollte nicht [lange, blonde, kluge] Frau heiraten?

die [heutzutage kaum noch zu bestehenden] Moralität.

\subsection{Die Erweiterung der Adjektivphrase}

Unter genanntem Begriff (Erweiterung), der mit komplexen Adjektivphrasen kommt, erscheinen die folgenden drei Phänomene (Vgl. Nach George 1995:244.299):

- Graduierung: bei diesem Erscheinung wird ein Adjektiv durch unflektierte Adjektiv oder Adverbien ändert:

Er ist [richtig einfach].

Die Blume wurde [ganz schön pink].

- Wortstellung: hier reihen mehrere gleichmäßige Adjektive aufeinander sowohl verbundene oder nicht unverbundene:

die [ausgestellten irakischen] Antiquitäten.

meine [alten und modernen] Schuhe.

- Erweiterung: Bei diesem Erscheinung wird Kern des Adjektiv sich um gesamte Phasen ausgeweitert:

ein zu [[dieser Zeit] [von jedem] geschätzten] Bildhauer.

\subsection{Die Graduierung der Adjektivphrase}

Bei der Graduierung ist der Fall, wenn Stufenweise keine Flexion hat, können mehrere Veränderungen möglich sein. Dies erledigt, wenn modifizierende Adjektiv oder Adverb vorangehen sein (Vgl. nach George 1995: 244-300):

sein wundersam früher Hausbesuch

sein vollends wundersam früher Hausbesuch

In Folgendem werden die unflektierten, modifizierenden Adjektive von die deklinierten, parallelen unterschieden:

$$
\begin{aligned}
& \text { der erwartet hohe Misserfolg } \\
& \text { der erwartete, hohe Misserfolg }
\end{aligned}
$$

Im ersten Fall ist der Misserfolg wahrscheinlich nicht erwartet, aber nur so hoch geschieht; im zweiten Fall war der Misserfolg auch erwartet.

Angeordnete Adjektive halten eine Reihung. Diese Art von Adjektiven stellen näher beim Substantiv, wenn ihrer Bedeutung einigermaßen Artikelwörtern benutzen. 


\subsection{Die Adjektivstellung}

Angeordnete Adjektive halten eine Reihung. Diese Art von Adjektiven stellen näher beim Substantiv, wenn ihrer Bedeutung einigermaßen Artikelwörtern benutzen; wenn sie eher Substantiven verwandt sind, werden sie dann näher beim Substantiv sein. Bezüglich gibt es drei Zonenbereichen (Vgl. nach George 1995: 254-301):

\begin{tabular}{|c|c|c|c|c|}
\hline & Bereich 1 & Bereich 2 & Bereich 3 & \\
\hline & Zahl/Hinweis & Darstellung/Werten & Art und Weise/Quelle & \\
\hline die & zwei & Schmackhaften & Wiener & Bratwürste \\
\hline \multirow[t]{3}{*}{ alle } & bekannten & Guten & Irakischen & Dattelbäume \\
\hline & & Bitter & Brauner & Kaffee \\
\hline & solcher & Roter & Wollenen & Pullover \\
\hline die & rückwärtigen & Schadhaften & Schadhaften & Zähne \\
\hline
\end{tabular}

In der ungeläufigen oder steifen Reihung wird das zweite Adjektiv als mehr eigenwertiges Adjektiv:

akademische, erhebliche Hindernisse

kriminelle, unbekannte Fakten

Die Ordnung der Adjektive übereinstimmt auch ihrer inhaltlichen Beziehung mit dem Substantiv. Wenn ein Adjektiv näher von dem Substantiv stellt, dann sie wird seine enge Beziehung inhaltlich ans Substantiv zu haben, auch die Einheit mit dem Substantiv so stärker wird. Gemäß was oben erwähnt, die Adjektive der Zonen im Vorwärts unterordnen diejenige, die der hinteren Zonen. Im Rahmen dieser Unterordnung gibt es auch keine Bindewörter zwischen Adjektiven, die in den unterschiedlichen Zonen steht, (Vgl. Grammatik und Stil 2005:246), so kommt nicht:

die nächsten und lächerlichen Kapitel

die beiden und regierenden Seiten

Man kann erschließen, dass durch ein Bindewort so oft die Bedeutung auch sich ändert. leckere und Italienische Pizza meint hier zwei Gattungen und vielleicht auch, dass die Italienische nicht als lecker eingestuft werden.

Gibt es anderem Fall, die bei Adjektive, die nebeneinander steht, die Reihung dient öfter der Steigerung.

der exotische, ja barbarische Mann

die wenigen, wahrscheinlich einmaligen Findung

Zusammenfassend kann man erschließen, dass Adjektive substantiviert werden können. Auch „, sie werden dann wie attributive Adjektive flektiert und wechseln nicht etwa zu einem substantivischen Flexionsmuster“" (Duden 2005:354-473).

\section{FAZIT}

Hier kann man schlussfolgern, dass die Adjektive semantisch unterschiedlich erweisend sind. Manche von ihnen reihen eine Eigenschaft einer Person oder Sache ein, hier spricht man von Adjektiven, die qualifizierende Eigenschaften haben. In dieser Forschung wurden im Allgemein die Phrasen untersucht, und sie erscheint als Zwischenglieder zwischen Wort und Satz. Diese Arbeit hat insbesondere gezeigt, dass Phrasen bestimmte Eigenschaften und Arten verfügen. 
In diesem Zusammenhang erschließen können, das Adjektiv zu Art und Weise des Textes passen sollte und es stilistisch und inhaltlich im Gegensatz zu seinem Referenzwort sein sollte. In der Adjektivphrase geht sie nahezu häufig dem Adjektivkern voraus, wie sie Kern des Adjektivs sich um gesamte Phasen ausgeweitert wird.

Die Adjektivphrasen können sich im Satz auf die Subjekt- oder Objektnominalphrasen oder das Verb bezieht. Die Adjektivphrase hat Funktion im Satz, sie kann als ein attributiv, adverbial oder Prädikativ verwendetes Adjektiv sein. Im Satz kommt die Adjektivphrase vornehmlich prädikativ vor. Im Satz kommt die Adjektivphrase vornehmlich prädikativ vor.

Es wird erklärt, dass das Adjektivsatz vor oder nach einem Nomen stellen kann, Adjektivphrasen, die vor dem Nomen erscheinen, werden als attributive Phrasen näher festlegt, während Adjektivphrasen, die nach dem Nomen erscheinen, werden Prädikativphrasen bezeichnet

Im eigentlichen Sinne die Adjektivphrase sind unterscheidet von den mit einem Partizip II gebildeten Phrasen. Adjektive und Adjektivphrase treten sehr häufig in Reihung nicht nur als Attribut sondern in der prädikativen Verwendung auf.

Man kann erschließen, dass durch ein Bindewort so oft die Bedeutung auch sich ändert. Adjektive können zu einer Adjektivphrase erweitert werden, indem sie vor Substativen auftreten und werden dann von diesen regiert oder mit einer Angabe (Sie können als Bauteile von Wortgruppen in einem Satz mit einem Nomen) modifiziert werden.

Abschließend werden die Ergebnisse der Forschung zusammengefasst, dass die Phrase Wortgruppe ist, die mit einander eine Sinneinheit konstituieren.

\section{LITERATUR:}

- Duden: Die Grammatik; unentbehrlich für richtiges Deutsch- 7., völlig neu erarbeitete und erweiterte Auflage: Herausgegeben von der Dudenredaktion/Dudenverlag: Mannheim. Leipzig. Wien. Zürich 2005.

- Duden, 2006, Die Grammatik-Band4, Duden Verlag, Mannheim.

- Eisenberg, Peter (1999). Grundriss der deutschen Grammatik. Bd. 2 Der Satz. Stuttgart/Weimar: Metzler, Kap. 13.2 „Zur Topologie der Nominalgruppe“, 400-410.

- George (den großen Grammatik) Grammatik und Stil: Praktische Grammatik des Deutschen; 1. Auflage - 2. Druck 1995, 1989 Cornelsen Verlag, Berlin-ISBN 3-454-22705-4- Bestellnummer 227054.

- Grammatik und Stil: Praktische Grammatik des Deutschen; 1. Auflage - 2. Druck 1995, 1989 Cornelsen Verlag, BerlinISBN 3-454-22705-4- Bestellnummer 227054.

- Heringer Hans, 1989, Grammatik und Stil, Cornelsen Verlag, Berlin.

- H. Leiß 7/98. Syntax I Skriptum einer 2-stundigen Vorlesung/Sommersemester 1998 Centrum für Informations- und Sprachverarbeitung/Universität München.

- Kürschne Wilfried, 2003, Grammatisches Kompendium, A. Franke Verlag, Tübingen und Basel.

- Wahrig: Deutsches Wörterbuch, Bertelsmann/Lexikon Institut; Wissen Media Verlag GmbH, Gütersloh/München 2005(vormals Bertelsmann Lexikon Verlag GmbH) Alle Rechte vorbehalten ISBN 3-577-10079-6.

\section{INTERNETQUELLEN:}

- $\quad$ https://grammis.ids-mannheim.de/progr@mm/4067, 28.9.2020, 12:00 A.M.

- $\quad$ http:// www.dwds.de, 28.10.2020, 12:00 A.M. 